Ingeniería Investigación y Tecnología, ISSN 2594-0732,

IX. 2. 171-181, 2008 (artículo arbitrado)

DOI: http://dx.doi.org/10.22201/fi.25940732e.2008.09n2.014

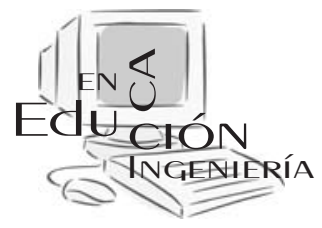

\title{
Los límites del pronóstico newtoniano y la búsqueda del ORdEN EN El CAOS
}

\section{The Limits of the Newtonian Forecast and the Search of ORdER IN THE CHAOS}

\author{
N. Sánchez-Santillán ${ }^{1}$, M.R. Garduño-López ${ }^{2}$, W. Ritter-Ortiz ${ }^{2}$ y S.A. Guzmán-Ruiz ${ }^{2}$ \\ ${ }^{1}$ Departamento el Hombre y su Ambiente, Universidad Autónoma Metropolitana, \\ ${ }^{2}$ Centro de Ciencias de la Atmósfera, UNAM, México \\ E-mails: santilla@correo.xoc.uam.mx,rene@atmosfera.unam.mx, \\ walter@atmosfera.unam.mx y sergio@atmosfera.unam.mx
}

(Recibido: noviembre de 2005; aceptado: febrero de 2007)

\section{Resumen}

La mecánica determinista newtoniana sólo puede describir y pronosticar el comportamiento de los sistemas naturales simples y con pocos componentes, aproximadamente el $10 \%$ del total de los que conforman la realidad universal conocida hasta ahora; el $90 \%$ restante, cuya complejidad y grado de incertidumbre los hace prácticamente inaccesibles a este enfoque, requieren de una nueva visión holística o total, con un acercamiento que incluya conceptos, tanto de la mecánica clásica de Newton y Descartes, como los emanados de la corriente indeterminista, entre los cuales se encuentran la no linealidad y las secuencias aleatorias, el cálculo de la probabilidad y la estadística, el caos y el orden, la inestabilidad exponencial, la teoría cuántica, los atractores y fractales y la teoría de la información.

Descriptores: No lineal, pronósticos, orden, caos, estocasticidad.

\begin{abstract}
Newtonian deterministic mechanichs can only describe and predict the behavior of simple natural systems with few components, which represent approximately $10 \%$ of those conforming the universal reality known until now. The remaining $90 \%$, whose complexity and degree of uncertainty make them practically inaccessible to this approach, require a new holistic or total vision, with an approach that includes concepts of Newton's and Descartes's classical mechanics, as much as those emanated from the indeterministic stream, such as nonlinearity and aleatory sequences, calculus of probability and statistics, chaos and order, exponential instability, quantum Theory, attractors and fractals, and information theory.
\end{abstract}

Keywords: Non-linearity, prognosis, order, chaos, stochasticity.

\section{Introducción}

Dos paradigmas científicos compiten en nuestros días por explicarnos las circunstancias y la posible evolución de los fenómenos que ocurren en la Tierra y el Universo: por un lado, el viejo y exitoso enfoque determinista, el cual, a partir del siglo XVII y con las ideas de Galileo, Descartes, Bacon y Newton (Sánchez-Santillán y Garduño, 2007), principalmente, ha sido la base de la mayoría de los logros en la ciencia y la tecnología; por otro lado, la visión indeterminista 
Los límites del pronóstico newtoniano y la búsqueda del orden en el caos

o estocástica, que desde finales del siglo XIX y hasta el presente, ha tenido que incorporar y conciliar, cuando ha sido posible, tanto el mecanicismo, como las nuevas teorías del conocimiento, incluidas la cuántica, la de la relatividad, la del caos, la de la probabilidad y la de la Información - por mencionar algunas- para poder explicar y predecir el comportamiento de la naturaleza en sus sistemas más simples, en los más complejos y aún en aquéllos en los que el azar pareciera ser la única base objetiva de sus conductas.

La simplicidad o complejidad de los sistemas está dado por el número de elementos que lo componen, las interacciones potenciales que establezcan y la cantidad de estados posibles en que estas últimas se traduzcan (Sánchez-Santillán y Garduño, 2007). La atmósfera, por ejemplo, es un sistema de una enorme complejidad, donde se observa que las regiones más alejadas terminan influyéndose recíprocamente. El carácter aleatorio de todo pronóstico parecería deberse sencillamente a la imposibilidad de dominar todos los parámetros significativos que definen su dinámica. Sin embargo, no por el hecho de que el sistema sea complicado, su comportamiento es imprevisible y, por el contrario, existen sistemas muy simples cuya conducta es también muy compleja. El papel que juega el azar ha ido cobrando relevancia conforme se profundiza en la naturaleza de los fenómenos, sin importar la diversidad de sus estructuras. Siguiendo este orden de ideas, es difícil sustraerse al pensamiento de que estaríamos mucho mejor si hubiese más gente, no sólo en la investigación sino también en el mundo de la política y la economía, que comprendiera que los sistemas simples no poseen, necesariamente, propiedades dinámicas simples, (Ritter et al., 1997).

Desde mediados del siglo XIX ya se sabía que, inclusive, algunos de los sistemas deterministas -aquellos en que el conocimiento de sus parámetros significativos permiten pronosticar con certeza su evolución (Sánchez-Santillán y Garduño, 2005 en prensa)- podían comportarse de un modo aparentemente aleatorio. Esto podía deberse a alguna información imperfecta sobre ellos y ocurría, principalmente, en los más grandes y complicados, con muchísimos grados de libertad (variables del sistema), y una enorme cantidad de partes constituyentes cuyo comportamiento detallado parecería estar siempre más allá de la capacidad de la mente humana para poderlos entender. Por otro lado, el progreso completo de la ciencia estaba basado en la convicción de que la forma de buscar simplicidad en la naturaleza era hallando ecuaciones diferenciales para describirla. La humanidad siempre se ha esforzado por comprender las regularidades de los fenómenos naturales y encontrar las leyes ocultas tras las complejidades del universo, es decir, por descubrir el orden que subyace en el caos. La realidad práctica es que la matemática constituye el método más efectivo y confiable de que disponemos para el entendimiento de nuestro entorno. Conforme una teoría suplanta a otra y un paradigma destrona a otro paradigma, una cosa permanece: la aplicabilidad de la matemática.

En este trabajo se ponderarán las características, alcances e implicaciones de los enfoques determinista e indeterminista, haciendo mención del tipo de matemáticas que cada uno de ellos ha desarrollado como herramienta indispensable para su propia justificación; se aplicarán algunos conceptos derivados de estas posturas a un sistema complejo en concreto, el atmosférico, y se introducirá el concepto de holismo o ciencia del todo, como una inevitable síntesis de enfoques con la cual es objetivamente imposible enfrentar con mayor competencia la comprensión y predictibilidad del universo.

\section{Determinismo y estocasticidad ambiental}

La visión determinista considera que todos los fenómenos se pueden explicar (Sánchez-Santillán y Garduño, 2007). El determinismo absoluto afirma que con un conocimiento perfecto de lo que un objeto hace en el presente, es posible predecir con exactitud lo que hará en cualquier momento del futuro, (Campbell, 1982), está íntimamente

172 Ingeniería Investigación y Tecnología, ISSN 2594-0732 
relacionado con la causalidad, noción que postula que los sucesos tienen una causa de la cual se generan efectos y donde al conjunto de eventos que los desencadenan se les denomina condiciones iniciales. Dada su mecánica determinista, el comportamiento futuro y pasado de cada hecho podría saberse con certeza; de acuerdo a Laplace, la totalidad de las cosas son predecibles con sólo saber las posiciones y velocidades de cada una de las partículas del universo.

La mecánica de Newton, con sus tres siglos de antigüedad, proporciona una teoría vital que sigue siendo utilizada en toda clase de disciplinas donde se elaboren y anticipen trayectorias -ya sea de planetas, misiles, satélites o huracanes- la cual funciona con increíble exactitud, razón por lo que sigue siendo de uso diario. Para describir conductas de sistemas sencillos que contengan un número muy pequeño de cuerpos en movimiento, la física de Newton sigue siendo muy útil; no obstante, este determinismo es a menudo irrelevante en una enorme cantidad de sistemas del mundo real donde es imposible poseer un conocimiento per- fecto de la forma en que se comportan, debido a su complejidad y a la gran cantidad de cuerpos que lo integran, (Ritter et al., 1997).

Para los filósofos, la idea de causalidad no es del todo clara ni está bien fundamentada y no resiste un punto de vista estrictamente lógico. La cadena de la causalidad lineal es una mezcla de costumbres, creencias y sentido común, cuya estructura inamovible es necesario cuestionar. A medida que la ciencia explora con más profundidad el universo de flujos internos y desarrollos dinámicos, de influencias sutiles y escalas de tiempo, las cadenas causales ya no se pueden analizar y reducir a conexiones lineales de sucesos individuales, de modo que el concepto mismo de la causalidad empieza a perder fuerza.

Por ejemplo, cuando la causalidad y el determinismo se utilizan en algo tan complejo como un sistema ecológico, las redes interrelacionales empleadas se vuelven tan intrincadas, que las teorías y los modelos matemáticos, empleados en un universo extremadamente causalista no funcionan. Sin embargo, el enfoque indeterminístico o estocástico trajo una nueva visión de las cosas, de las cuales sus repercusiones apenas han comenzado a socavar los cimientos de la física clásica, echando abajo las ideas sobre la conexión de los acontecimientos.

Fue Willard Gibbs el primero en reconocer que el universo es predecible sólo dentro de los límites estadísticos, es decir, no podemos seguir ocupándonos de lo que siempre ocurre, sino de lo que ocurre en una abrumadora mayoría de las veces. Todos los sistemas pueden ser descritos en forma estadística. La aparente causalidad del universo se debe al hecho de que las probabilidades en los sistemas mayores, más que en los pequeños, son aproximadamente iguales a uno. El azar no es un nuevo instrumento matemático aplicable a la física, sino parte esencial de su entramado, y la estocasticidad, palabra que se deriva del griego stokos que significa adivinar (Campbell, 1982), es el acercamiento gnoseológico que reconoce lo fundamental de esta condición. Ello implica que la apariencia de la causalidad en la vida cotidiana es un puro engaño estadístico y que todas las realidades posibles coexisten sin dejar fuera aún aquellas inaceptables para las propias instituciones. Vivimos en un universo perceptivo.

La naturaleza debe ser interpretada como el campo de acción de la materia, de la energía y de la información, presentando, junto con sus formas de vida, una base azarosa - no necesariamente accidental- donde las fuerzas del azar y del no-azar coexisten en una relación complementaria. Así, Einstein descubrió que el movimiento Browniano (movimiento errático, de agitación constante, observada en partículas diminutas de polvo) presenta frecuentemente en el universo. Su matemática representativa está basada no en una relación de causa y efecto, sino en el azar y la estadística, introduciendo con esto una nueva manera de ver y analizar los fenómenos naturales (Campbell, 1982). 
Los límites del pronóstico newtoniano y la búsqueda del orden en el caos

También Wiener (1948) logró reconocer que el envío y recepción de mensajes en las comunicaciones tiene bastantes cosas en común con el movimiento Browniano. El lazo que une a estos dos aspectos es la estadística, una rama de la teoría de la probabilidad, de esta última se dice que es el principio maestro tras algunos de los más profundos descubrimientos de la física moderna. Las sucesiones de estos hechos espaciados en el tiempo, cuando no son de antemano y por completo conocidos, se les denominan secuencias estocásticas. Al utilizar este tipo de matemáticas en la predicción, no se está tomando en cuenta un solo futuro, sino muchos futuros simultáneos que, pueden considerarse, coexisten en sentido abstracto. Aquí, la estadística no puede hacer nada con un solo dato, ya que un hecho aislado carece de sentido. Es necesario ser parte de un modelo de muchos hechos posibles en el que cada uno tenga cierta posibilidad de ocurrir (Campbell, 1982).

Wiener (1948) expuso su firme convicción de que un científico nunca podrá tener un conocimiento perfecto de lo que una parte del universo hace mientras se le observa en determinado momento. Como consecuencia, no hay esperanza de conocer con seguridad y en detalle lo que será en el futuro. Gerolamo Cardano se percató además de que la incertidumbre posee una estructura y de que una vez descubiertas sus claves, el azar deja de ser nebuloso y se vuelve inteligible (Campbell, 1982).

Sin embargo, la complejidad del mundo es ilimitada y las partes de éste se encuentran en continuo cambio. De nada de lo que se pueda afirmar sobre él en un momento dado, puede decirse que es absolutamente cierto. La teoría de la información de Shannon y Weaver (1949) se ocupa de este tipo de errores como algo intrínseco y fundamental, y las probabilidades sólo pueden existir como resultado de los datos que arrojen los experimentos. Es imposible hablar de la probabilidad de un hecho aislado: tiene que haber muchos. El experimento debe continuar hasta que exista una razón de peso para creer, de manera confiable, que la frecuencia relativa se aproxima a un límite fijo si se prosiguiera indefinidamente. El valor de este límite es la probabilidad.

La probabilidad que se encuentra en la raíz de la teoría de la información, está relacionada con la cantidad y el tipo de conocimiento que poseemos sobre cualquier serie de hechos, cuyo desenlace es incierto. El científico debe tratar de superar esta incertidumbre sobre su compromiso futuro, considerando una gama de diferentes contingencias y asignando una probabilidad apropiada a cada una: entonces se puede señalar lo que quizá suceda en tal o cual conjunto de circunstancias. Estos nuevos conceptos de comunicación y control conllevan una interpretación diferente del hombre, del conocimiento, del universo y de la sociedad (Wiener, 1948).

\section{Los alcances de predicción de la física newtoniana y la situación actual del pronóstico meteorológico}

El modelo mecanicista de Newton y Descartes está caracterizado por el materialismo y el inductivismo, enfoca la mirada más sobre las cosas que sobre sus relaciones, lo cual implica que el universo material es como una máquina que puede entenderse completamente con sólo analizar por separado sus partes más pequeñas. Para que así ocurra es necesario que los sistemas estudiados se comporten de manera lineal, es decir, al sumar sus componentes, el resultado exprese la totalidad del fenómeno (Sánchez-Santillán y Garduño, 2007).

Sin embargo, aproximadamente un $90 \%$ de los sucesos de la naturaleza se comportan justamente como sistemas no-lineales, en los que el origen y resultado divergen y donde los algoritmos lineales poco o nada pueden hacer para descifrarlos.

Desafortunadamente para los meteorólogos clásicos actuales, la atmósfera es un sistema complejo no lineal donde la más leve incertidumbre en la descripción de las condiciones iniciales conducirá a futuros muy distintos. Liquidándose de

174 Ingeniería Investigación y Tecnología, ISSN 2594-0732 
una vez por todas el sueño determinista en la meteorología y, aunque se utilizan modelos matemáticos cada vez más laboriosos e ininteligibles, los sistemas no-lineales sólo pueden ser descritos por la interacción de sus variables y no por la mera acción de ellas, cambiando drásticamente ante variaciones mínimas en sus componentes.

El pronóstico del tiempo se sirve de varias herramientas metodológicas y matemáticas. Algunas de las más comunes son: las tendencias de la temperatura, la humedad y la presión atmosférica; la comparación con situaciones similares en el pasado con sus tendencias a largo plazo; las teleconexiones, las ecuaciones de regresión y las funciones empíricas ortogonales; las contingencias climatológicas, que indican probabilidades de ocurrencia después de observadas ciertas condiciones atmosféricas; el uso de anomalías en la cubierta de nieve, de la temperatura superficial oceánica y de la humedad del suelo para determinar probables patrones de forzamiento y estabilización. La correlación de éstas y muchas otras variables, mediante algoritmos cada vez más complejos y con la ayuda imprescindible de poderosísimas computadoras, se da paralelamente a la creación y perfeccionamiento de métodos numéricos, siempre adecuados y confiables.

Las ecuaciones que rigen la circulación atmosférica, de las que depende el clima, tienen la propiedad de la inestabilidad exponencial, en donde a una condición inicial dada de cierto estado de la atmósfera (presión, temperatura y humedad) corresponde una evolución futura perfectamente determinada, resultado de un cálculo en el que el azar no interviene.

Si modificamos ligeramente esta condición inicial, tan sólo este cambio ínfimo tendrá pequeñas consecuencias en los primeros instantes, pero experimentará una tendencia a amplificarse en el tiempo a un ritmo exponencial. Realmente, si se pretende prever lo que va a ocurrir, es menester tener en cuenta todo lo que nos rodea (Ritter et al., 1997).
Von Neumann (1949) advirtió las limitaciones de esta manera de encarar las cosas y comprendió que la índole de las ecuaciones en relación con las condiciones iniciales impediría siempre todo pronóstico a largo plazo; pero llegó también a una conclusión más original: la posibilidad de que esa misma inestabilidad permitiera dirigir el tiempo; acaso fuera más fácil orientarlo que preverlo. La inestabilidad exponencial y la dificultad de predecir, lo que es su consecuencia, son fenómenos corrientes que se manifiestan en las situaciones más variadas, tanto en las más simples como en las más complejas. El tiempo que se podrá observar dentro de un año revelará información sobre el estado de la atmósfera prevaleciente el día de hoy, a excepción de aquellos datos en una escala demasiado fina que no sea posible percibirlos directamente. Sin embargo, es más práctico considerar que hay creación de información con el correr del tiempo y decir que la evolución futura del sistema depende del estado actual y del azar.

En tanto qué condiciones geográficas semejantes producirán efectos climáticos semejantes, no es cierto que condiciones atmosféricas semejantes, produzcan condiciones geográficas semejantes. No obstante, aunque la inestabilidad exponencial impide toda previsión cuantitativa a largo plazo, no excluye previsiones de orden cualitativo, incluso en plazos mucho mayores.

Del mismo modo que en el siglo XVIII se creía en un mundo que funcionaba como un reloj, a mediados del siglo XX se creía en uno no lineal. La Teoría del Caos, al estudiar estructuras irregulares presentes en el mundo natural, ha descubierto que en ciertos sistemas deterministas se producen comportamientos que, en su aparente aleatoriedad, son completamente impredecibles.

Esta teoría, que empezó a desarrollarse hacia finales del siglo XIX con el físico-matemático Henri Poincaré (1944), introductor del concepto de nolinealidad, encuentra que mientras más se detalle un problema, su solución se vuelve más difusa; las estructuras más complejas tienen propiedades di- 
Los límites del pronóstico newtoniano y la búsqueda del orden en el caos

ferentes a las de sus componentes más simples, por lo que se pierde la capacidad de predicción.

Es así que a partir de los conceptos de nolinealidad, de inestabilidad exponencial, del papel del azar y las consecuencias aleatorias, del cálculo de la probabilidad y de la estadística, la estocasticidad se adentra en la interpretación de las cosas, tratando de salvar las fronteras de las distintas disciplinas, aceptando las paradojas que hacen inseparables a lo simple y lo complejo, al orden y al caos, buscando la armonía entre lo fijo y lo aleatorio, apuntalada con la visión que define al caos como una suerte de orden implícito (Sánchez-Santillán y Garduño, 2007).

Actualmente, la ciencia muestra que la naturaleza es inexorablemente no lineal. La matemática clásica se concentró en las ecuaciones lineales por un motivo lógico: no se podía resolver ninguna otra cosa. La matemática de los procesos estocásticos floreció después de la matemática de los procesos determinísticos. Dos formas de ver al mundo, dos paradigmas, dos ideologías matemáticas: el determinismo para los sistemas simples con pocos grados de libertad, la estadística para los complicados con muchos grados de libertad. Parecería plantearse una disyuntiva insalvable: los sistemas son aleatorios o no aleatorios.

Sin embargo, es posible observar como cada vez se estrechan los campos de acción del determinismo al surgir los elementos estocásticos de cada sistema. Por ejemplo, para poder hacer la predicción dinámica de un evento clásico como "El Niño", es necesario limitar, tanto los parámetros de las leyes físicas que intervienen en la interpretación del fenómeno, como las condiciones iniciales en que éste se produce, y todo con el propósito de hacer manejable el caudal de información que implica a los elementos del suceso y a la infinidad de interrelaciones que establecen.

Lamentablemente, debido al desconocimiento que aún en la actualidad se tiene de este evento, algunos de los parámetros y condiciones iniciales que se hacen a un lado pueden ser justamente los que sean críticos en su comportamiento, lo cual limita la exactitud de los pronósticos.

Por esta razón, los modelos que se usan en los servicios meteorológicos no arrojan buenos resultados y, por lo tanto, hay que desarrollar modelos de sistemas no lineales para este tipo de predicción, considerando que el sistema acoplado océano-atmósfera se caracteriza por la presencia de pequeñas diferencias en las condiciones iniciales que nos conducirán posteriormente a situaciones por completo diferentes, característica de un sistema caótico.

Sigue siendo un reto gigantesco formular una teoría completa de todos los supuestos en los cuales puede emerger el caos. No obstante, el determinismo macroscópico que impera en la escala de la meteorología, es reducible al azar cuántico gracias a las leyes de la estadística que se aplican a la presencia de grandes cantidades de partículas. Así, el azar parece ser el dato fundamental en la naturaleza.

\section{Atractores y fractales}

Para poder visualizar el comportamiento de los sistemas -los simples, los complejos y los caóticos- se utiliza el concepto de atractores, herramienta que muestra los conjuntos de valores más o menos regulares hacia donde tienden las dinámicas de aquellos. La graficación de los sistemas implica un modelo matemático de coordenadas llamado espacio de fases, constituido por las variables que describen al fenómeno estudiado y donde cada punto en este espacio representa uno de los estados posibles (Sametband, 1999).

Los atractores se mueven en una región del espacio de fases hacia la cual siempre convergen; pueden ser predecibles, como los llamados de punto fijo o los de ciclo límite, que corresponden a conductas periódicas en los sistemas y cuyo ejemplo más sencillo es el movimiento de un péndulo, el cual exhibe un atractor de ciclo límite al alcanzar

$176 \quad$ Ingeniería Investigación y Tecnología, ISSN 2594-0732 
su máxima extensión en ambos lados de su eje vertical y otro atractor de punto fijo, que es donde reposa al cesar su acción (Sametband, 1999).

Los atractores extraños o caóticos son aperiódicos; muestran un comportamiento completamente aleatorio; sin embargo, se mueven dentro de una región definida del espacio de fases, lo cual evidencia una cierta regularidad y posible orden dentro del caos aparente.

Algunos de ellos, pueden tener la estructura de un fractal, (cuando son de dimensión no entera), forma geométrica autorreplicable en cualquier escala en la que se observe. Las ecuaciones que describen los sistemas caóticos, procesadas iterativamente por computadora, originan una geometría fractal de dimensión fraccionaria (Gleick, 1991); generando gráficos parecidos a: árboles, montañas, nubes, bronquios, etc., es decir, a configuraciones naturales de las cuales hasta hace poco era impensable poderlas representar matemáticamente.

\section{Consideraciones en torno a nuevas metodologías de pronóstico}

Pronosticar el estado del tiempo es una cosa; pronosticarlo correctamente es otra. El principal defecto de los métodos actuales de predicción meteorológica es que no son muy eficientes en prevenir cambios repentinos; dicho de otra forma: es posible pronosticar siempre y cuando no ocurra nada inesperado.

A la función que describe el comportamiento estocástico de un sistema no lineal se le denomina logística. La aplicación de la ecuación logística no sólo pone de manifiesto la aparición del caos, sino también la manera en que puede ser creado y muestra que los cambios drásticos no tienen necesariamente causas drásticas.

Para estudiar la dinámica de esta ecuación se debe contemplar el comportamiento de sus atractores a largo plazo.
El mismo ordenamiento no sólo es válido para la aplicación logística, sino también para las iteraciones de cualquier aplicación de curvas de tipo unimodal. Los modelos del caos pueden ser universales, es decir, no específicos para ejemplos individuales, sino representativos de clases enteras de sistemas.

La cadena lineal de la causalidad y el poder profético de sus ecuaciones matemáticas han dominado la ciencia durante tantas generaciones, que ahora es difícil ver donde hay lugar para la libertad, la novedad y la creatividad. Si se examina detalladamente cualquier fenómeno resultará que el todo es la causa de las demás partes y sus efectos más sutiles adquieren cada vez mayor importancia. Al afirmar que el todo causa todo lo demás, se da a entender que los fenómenos de la naturaleza se originan en los cambios frecuentes del conjunto y se describen mejor con una ley del todo u holística. Mientras que la causalidad lineal puede funcionar adecuadamente para sistemas limitados, mecánicos y aislados, se necesita algo más delicado y complejo para describir la extensa variedad de la naturaleza.

Existe una economía en el universo; el movimiento y el cambio de él como un todo, Lagrange, Fermat y Euler demostraron que estos elementos se presentan de un modo que minimizan la acción de la naturaleza. Max Planck creía que los principios de variación eran generales y que debían aplicarse a todo tipo de comportamiento dentro del universo; por lo mismo, sólo podrían entenderse desde una perspectiva total.

La teoría de la relatividad y la teoría cuántica son consecuencia lógica de los principios universales de variación. Esta dinámica demuestra que todo movimiento y cambio surgen de una nueva ley del todo, y que los patrones y sucesos de la naturaleza son la expresión de una unidad fundamental de forma. La ciencia es un intento de comprender las cosas; es un planteamiento objetivo en que nada se da por cierto, la razón y el experimento son inseparables, contemplados den- 
Los límites del pronóstico newtoniano y la búsqueda del orden en el caos

tro del contexto de una percepción directa de la totalidad de los fenómenos y su unidad. Todo lo que ocurre en nuestro universo es causado por todo lo demás, aunque su totalidad se revela o se expresa en sus acontecimientos individuales. Las ciencias naturales acabarán englobando la ciencia del ser humano y, al mismo tiempo, la ciencia del ser humano englobará las ciencias naturales. No habrá mas que una ciencia, este es el fundamento del enfoque holístico.

Hace falta una visión generalista para darse cuenta de lo que es realmente importante; por otro lado, hoy en día existen signos de una nueva unificación de las matemáticas. Tradicionalmente las ciencias buscan estructuras y regularidades en los datos en que hay probabilidad de encontrar algún sentido en ello. Así, uno de los problemas principales que enfrenta la ciencia actual consiste en diferenciar el caos determinista del estocástico.

El caos estocástico, como sinónimo de desorden (caos desordenado, según Favre, et al., 1995), se caracteriza por una duración nula de la memoria estadística; en cambio, el caos determinista tiene una memoria estadística de cierta duración y puede ser representado por un sistema cerrado de ecuaciones, sometido a determinados límites y condiciones iniciales. Estos sistemas se definen como un fenómeno dinámico que hace que un sistema, en ausencia de ruido y de todo tipo de fluctuaciones internas y externas, presente un comportamiento aperiódico (Montero y Morán, 1993).

Tanto el orden como el caos determinista proceden de la misma fuente: los sistemas dinámicos descritos por ecuaciones diferenciales no lineales. Los regímenes ordenados muy a menudo son más importantes para la biología y la vida misma que los caóticos. La evolución caótica refuta cualquier predictibilidad a largo plazo y las medidas registradas en un experimento no pueden repetirse nunca. Sin embargo, el caos puede seguir siendo interpretado en términos del concepto de atractor. Aunque las ecuaciones diferenciales de los sis- temas irreversibles y de su evolución en el tiempo sean determinísticas, su sensibilidad anula el ideal de lo exacto y lo predecible. La más ligera incertidumbre, que siempre habrá en el mundo real, da al traste con la predicción.

El atractor extraño contiene una infinidad de posibilidades, aunque confinadas en una región finita; estos sistemas adoptan configuraciones diferentes a medida que pasa el tiempo y sin repetirse nunca, lo cual significa que pueden crear formas hasta el infinito. Un atractor extraño caótico es completamente distinto a los atractores de punto fijo y de ciclo límite, aunque también es estable pero, contrario a estos últimos, observa una enorme sensibilidad respecto a las condiciones iniciales. El comportamiento a largo plazo de un sistema atrapado por un atractor extraño depende, hasta los detalles más pequeños, de cómo empezó $y$, a diferencia de los de ciclo límite y punto fijo, es un objeto fractal, presentando una invariancia de escala, ya que su forma es la misma sin importar la escala en que se contempla el objeto.

El descubrimiento de los fractales ha revelado una nueva forma de contemplar niveles infinitos de complejidad de la naturaleza. En el caos resultante de ecuaciones dinámicas determinísticas no lineales, se generan internamente propiedades intrínsecas, diferenciándose de los efectos incontrolados del azar y de las fluctuaciones estocásticas.

Si utilizamos con mayor rigor y profundidad de análisis al observar a los sistemas vivientes, se empiezan a entender nuevas maneras de interpretar sus fluctuaciones y cambios, en donde el orden y la forma son creados por la presencia y guía de unos pocos principios y fórmulas y no mediante controles complejos; no obstante, en la búsqueda de nuestros orígenes estos principios adquieren una dimensión que ninguna fórmula puede captar, ya que la vida resultante de la evolución de la materia no es fruto del azar y tampoco se puede afirmar que la materia inventa, que la naturaleza fabrica o que el universo sabe. 
N. Sánchez-Santillán, M.R. Garduño-lópez, W. Ritter-Ortiz y S.A. Guzmán-Ruiz

Existen procedimientos sencillos para conducir estas organizaciones con menores esfuerzos y tensiones, ya que la naturaleza, a través de los fractales, nos enseña cómo crear diversas e intrincadas estructuras mediante tan solo unos pocos principios básicos. Por ejemplo, se piensa que las espirales, tan comunes en el universo, describen una forma general y profunda de cambio que llega a la disipación y luego a un nuevo ordenamiento, y que tales configuraciones son el resultado de una sabiduría colectiva manifestada en los fenómenos, que expresa intuiciones de totalidad, orden, simplicidad, azar y previsibilidad al buscar el entrelazamiento y desarrollo de las cosas.

A través de la vía conceptual y de la teoría cuántica, está emergiendo una nueva representación del mundo que sintetiza y sobrepasa a las corrientes filosóficas anteriores. Este concepto expresa una visión contraria al sentido común, pues nos sitúa más allá de los lenguajes y el pensamiento, por lo que presenta límites al saber, a través de fronteras físicas difícilmente franqueables, que acotan la realidad. Como muestra está la constante de Planck o quantum de acción, que señala el límite último de toda divisibilidad de la radiación, concepto por el cual la longitud y el tiempo de Planck se convierten en el intervalo de espacio y tiempo más pequeño posible de identificar y medir.

Las fronteras establecidas por la teoría cuántica indican que la realidad está velada y se encuentra más allá de nuestro alcance, destinada a permanecer así por mucho tiempo. Frente a esto, la objetividad y el determinismo simplemente no existen; la visión materialista desaparece y penetramos en un mundo totalmente desconocido. Asimismo, descubrimos que acontecimientos, en apariencia desordenados e imprevisibles, contienen en sus entrañas un profundo y sorprendente orden.

La teoría cuántica nos dice que para comprender la esencia del universo debemos sopesar conceptos tales como: la materia no es sólida ni concreta; una misma partícula puede ser detectada en dos sitios a la vez; la realidad fundamental no es físicamente accesible; el tiempo y el espacio son ilusiones. La existencia de estas verdades trascienden categoría de tiempo y espacio ordinario a través de una naturaleza que posee propiedades muy difíciles de captar, donde la realidad no es local ni causal y cuyas consecuencias de transformación superan cualquier experiencia e intuición.

El mundo que hoy conocemos es la expresión de una geometría quebrada y es de esta imperfección de donde ha podido surgir la vida. Del torrente de energía que fluye por el planeta, sin metas ni objetivos aparentes, se puede esparcir conciencia y vida al combinarse moléculas, dentro del mismo corazón del caos, para formar estructuras estables que constituyen los primeros ladrillos de la materia viva. Aquí, la única diferencia de fondo entre lo inerte y lo viviente es, simplemente, que uno es más rico en información que otro. Aún no se sabe si su presencia es fruto del azar o de una necesidad de la naturaleza; lo que sí se conoce es que la vida extrae sus propiedades de la tendencia de aquélla a organizarse y escalar hacia estadios más ordenados y complejos.

Para intentar saber más de nuestro mundo se debe abandonar las certidumbres de sus leyes y admitir que el universo no sólo es más extraño de lo que pensamos, sino más extraño aún de lo que podemos pensar. En su inmensa complejidad y a pesar de sus apariencias hostiles, el universo está hecho para engendrar orden y vida, inteligencia y conciencia. Así, al contemplar un pequeño copo de nieve se puede observar su sometimiento a sutiles leyes físicas y matemáticas, generadoras de ordenadas figuras únicas e irrepetibles. Su forma final contiene la historia de todas las condiciones atmosféricas que ha atravesado y recupera al mismo tiempo un orden y un equilibrio de las fuerzas de estabilidad e inestabilidad y de las interacciones resultantes de ellas a micro y macro escala.

Se podría pensar que el universo en sus diferentes manifestaciones no contiene azar, sino 
Los límites del pronóstico newtoniano y la búsqueda del orden en el caos

diversos grados de orden cuyas jerarquías es preciso descifrar; también, que este carácter aleatorio sólo representa la incapacidad de la mente humana para comprender los estadios superiores del orden. A partir de la teoría cuántica se puede describir y pronosticar con gran precisión el comportamiento de grupos de partículas, aunque no existe actualmente ningún medio de predecir acontecimientos individuales, es decir, no existe ninguna herramienta determinista de pronóstico que permita definir el proceso de selección para saber o decir qué partícula en lo individual se manifestará y cómo lo hará. Sin embargo, bajo el desorden visible de estos fenómenos existe una estructura ordenada profunda que puede hacer posible la especificación de leyes para explicar lo que interpretamos como azar, a pesar del carácter aleatorio del comportamiento individual, que encubre la organización del conjunto.

Ciertos fenómenos caóticos con movimientos aparentemente indescriptibles e imprevisibles se canalizan dentro de estructuras sobre un modelo subyacente denominado atractor extraño, el cual se desarrolla en un espacio de fases que contiene todas las informaciones dinámicas y todos los estados posibles de un sistema, según órbitas precisas que ordenan en profundidad el comportamiento de aquél.

Einstein consideraba que el carácter estadístico de la naturaleza sólo es aplicable a los problemas microscópicos, mientras que en el mundo macroscópico el determinismo seguía imperando como regla.

No obstante, la condición estadística se acepta cada vez más como una característica general de los sistemas dinámicos inestables, en los que se puede observar que sea cual fuere la precisión que posea respecto de sus condiciones iniciales, sólo se puede predecir a través de las probabilidades el hecho de que se produzca una de sus muchas estructuras posibles. En conclusión, no se sabe lo suficiente de las leyes de la naturaleza como para ser capaces de predecir su desarrollo posterior.
La no-linealidad es el rasgo característico de la evolución de los fenómenos naturales, en donde se encuentran los sistemas de no equilibrio. Las transformaciones del estado del tiempo, de las especies biológicas o de los sistemas ecológicos, son sólo una variante de sistemas complejos que surgen del flujo constante de la energía solar en la biosfera y que se desenvuelven a través de múltiples bifurcaciones en las que se intercalan largos períodos de estabilidad con oscilaciones aparentemente azarosas en épocas de inestabilidad. En los espacios de transición se encuentra el punto de criticalidad, llamado también borde del caos o fase de creatividad (Borjón, 2002). Se llega así a reconocer en todo esto una tendencia general de largo plazo y una direccionalidad de manifestación total. La interacción de caos y orden, refleja de esta manera el proceso evolutivo de la naturaleza y la heterogeneidad a pequeña escala puede generar un orden a gran escala.

Los sistemas fuera del estado de equilibrio se manifiestan como sistemas complejos que evolucionan hacia estados crecientes, tanto de tamaño, de complejidad, dinamismo y niveles más elevados de organización, como de una más estrecha interacción con el ambiente físico, el clima, considerado altamente complejo.

Las respuestas a los cambios desestabilizadores se manifiestan a través de saltos e impulsos comparativamente bruscos y son importantes para entender los patrones de evolución en los diversos dominios de la naturaleza que, al estar más alejados del equilibrio termodinámico, conllevan mayor sensibilidad de respuesta al cambio de sus estructuras, con más sofisticación de los ciclos y procesos de retroalimentación que los mantiene.

Las bifurcaciones catastróficas nos dan apariciones y desapariciones súbitas de atractores estáticos, periódicos o caóticos y son la clase de transformaciones que sustentan la evolución de sistemas que van desde los átomos, hasta las especies, incluidos los ecológicos, los climáticos o los que se generan en las sociedades. 


\section{Referencias}

Borjón N.J. Caos, orden y desorden en el sistema monetario y financiero internacional. Plaza \& Valdés. México. 2002.

Campbell J. Grammatical Man: Information, entropy, language and life. Simon \& Schuster Press. Nueva York. 1982.

Favre A., Guitton J. and Lichnerowicz A. Chaos and determinism. Turbulence as paradigm for complex systems converging towards final states. (translated by Schwarzbach B.E.). The Johns Hopkins University Press. Baltimore and London. 1995.

Gleick J. Chaos: Making a new science. Viking Penguin Press. New York. 1991.

Montero F. y Morán F. Biofísica. Procesos de autoorganización en biología. Eudema Press. Madrid. 1993.

Poincaré H. Ciencia y método. Espasa-Calpe. Argentina. 1944.

Ritter W. Klimek R y Patiño R. Sobre el pronóstico en los sistemas físico-climáticos y biólogicoproductivos, posibilidades y limitaciones. Revista Geográfica-I.P.G.H, (123): 203-218. 1997.
Sametband M.J. Entre el orden y el caos, la complejidad. Serie: La ciencia para todos. Fondo de Cultura Económica, SEP y CONACYT. $2^{a}$ ed. México. 1999.

Sánchez-Santillán N. Y Garduño-López R. El clima, la ecología y el caos desde la perspectiva de la teoría general de sistemas. Ingeniería Investigación y Tecnología, (3): 183-195. 2007.

Shannon C.E. and Weaver. The mathematical theory of communication. University of Illinois Press. 1949.

Von Neuman J. Conference held at University of Illinois. In: Arthur W. Burks, (comp.) 1966. Theory of Self-Reproducing Automata Ur- ban. University of Illinois Press. 1949.

Wiener N. Cybernetics, or control and communication in the animal and the machine. Wiley \& Sons. New York. 1948.

Wiener N. I am a mathematician; the later life of a prodigy; an autobiographical account of the mature years and career of norbert wiener and a continuation of the account of this childhood in Ex-prodigy. Mass., MIT Press. Cambridge. 1966. 
DOI: http://dx.doi.org/10.22201/fi.25940732e.2008.09n2.014

Los límites del pronóstico newtoniano y la búsqueda del orden en el caos

\section{Semblanza de los autores}

Norma Sánchez-Santillán. Realizó sus estudios en hidrobiología en la Universidad Autónoma Metropolitana, plantel Iztapalapa. Obtuvo el grado de maestra en ciencias (Biología), orientada en aspectos de climatología en la Facultad de Ciencias de la UNAM en 1994. Posteriormente, alcanzó el grado de doctora, siguiendo la línea de aplicación climática en aspectos de ecología (1999). Cuenta con un diplomado en divulgación de la ciencia, por parte de la Dirección General de Divulgación de la Ciencia, UNAM (2004). Es autora de 70 trabajos publicados en revistas con arbitraje; así como 55 artículos de divulgación en revistas arbitradas. Pertenece al Sistema Nacional de Investigadores desde el año 2002. Actualmente se desarrolla como investigadora en la UAM-Xochimilco y como responsable del Laboratorio de Climatología, así como profesora dentro del plantel.

Manual René Garduño-López. Tiene licenciatura en física y posgrado en geofísica por la Facultad de Ciencias de la UNAM, en donde es profesor desde 1976. Es investigador titular del Centro de Ciencias de Atmósfera (CCA), UNAM, en la línea de cambios climáticos naturales y antropógenos. Ha publicado 24 artículos de investigación en revistas arbitradas y siete capítulos en libros, asimismo ha presentado 30 trabajos en congresos internacionales y 46 en nacionales. Es editor asociado de la revista Atmósfera (incluída en Science Citation Index) y ha editado la obra completa de Julían Adem, publicada por El Colegio Nacional en cinco volúmenes. Es autor de los libros El veleidoso clima y Pormenores terrestres, del Fondo de Cultura Económica. Fue Subdirector de la Dirección General del Personal Académico (UNAM); en el CCA ha sido Secretario Académico y actualmente es jefe de departamento.

Walter Ritter-Ortiz. Es doctor en biología con la especialidad en ecología y medio ambiente (UNAM). Realizó su licenciatura en física y matemáticas (UAG) y la maestría en ciencias geofísicas con la especialidad en climatología (UNAM). Es investigador en el Centro de Ciencias de la Atmósfera (UNAM) y jefe de la sección de Bioclimatología. Actualmente su área de especialidad es la simulación y manejo de recursos naturales, así como el cambio climático y la dinámica de sistemas.

Sergio Antonio Guzmán-Ruiz. Es investigador de la sección de Bioclimatología del Centro de Ciencias de la Atmósfera, UNAM. Cuenta con más de 30 artículos publicados en revistas nacionales e internacionales en áreas de teoría del clima, productividad oceánica y clima, productividad terrestre y clima, microclimatología, cambio climático y dinámica de sistemas. 